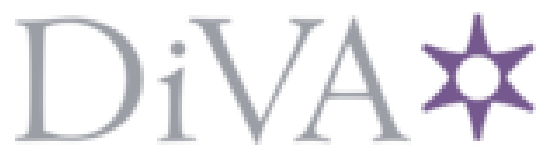

http://www.diva-portal.org

\title{
Postprint
}

This is the accepted version of a paper presented at 1oth IEEE International Conference on Intelligent Systems, IS 202O, Sofia, Bulgaria, 28 August 2020 through 30 August 2020.

Citation for the original published paper:

Sai Vineeth, V., Kusetogullari, H., Boone, A. (2020)

Forecasting Sales of Truck Components: A Machine Learning Approach

In: Sgurev V.,Jotsov V.,Kruse R.,Hadjiski M. (ed.), 2020 IEEE 1oth International

Conference on Intelligent Systems, IS 2020 - Proceedings, 9200128 (pp. 510-516).

Institute of Electrical and Electronics Engineers Inc.

https://doi.org/10.1109/IS48319.2020.9200128

N.B. When citing this work, cite the original published paper.

Permanent link to this version:

http://urn.kb.se/resolve?urn=urn:nbn:se:bth-20602 


\section{Forecasting Sales of Truck Components: A Machine Learning Approach}

\author{
Venishetty Sai Vineeth \\ Department of Computer Science \\ Blekinge Institute of Technology \\ Karlskrona, Sweden \\ Email: vesi17@student.bth.se
}

\author{
Huseyin Kusetogullari \\ Department of Computer Science \\ Blekinge Institute of Technology \\ Karlskrona, Sweden \\ Email: huseyin.kusetogullari@bth.se
}

\author{
Alain Boone \\ Director MAS, E2E Portfolio \\ Volvo Group \\ Gothenburg, Sweden \\ Email: alain.boone@volvo.com
}

\begin{abstract}
In this paper, forecasting sales model for truck components using machine learning algorithms is proposed. The forecasting model helps companions (i.e. Volvo Trucks) in the activity of trade and business. It also plays a major role for firms in decision-making operations in the areas corresponding to sales, production, purchasing, finance, and accounting. In order to achieve good forecasting sales mode, firstly, a normalization approach is performed on the time-series data to reduce and eliminate the data redundancy. After that, feature extraction and selection techniques are employed on the normalized data. Finally, different machine learning methods such as Support Vector Machine Regression, Ridge Regression, Gradient Boosting Regression and Random Forest Regression have been applied to the features of the normalized time-series data. Results depict that ridge regression method gives the most promising forecasting sale results of truck components compared to the other machine learning methods.
\end{abstract}

Keywords-Machine learning; time-series data; normalization; sales forecasting; feature extraction and selection.

\section{INTRODUCTION}

Good forecasts play a vital role in many fields of scientific, industrial, commercial and economic activity [4]. In today's business world's consumer-centring environment, companies requiring good sales results often need to provide trade-off a balance between customer demand and controlling cost of inventory. Maintaining broader inventory may allow customer demand to be satisfied at most of the times, but may lead to over-stocking, leading to major problems such as tied-up capital, and lower profit margins. In similarity, lower inventory quantities can reduce the inventory expenses but can occur in cost of chance arising from missed sale opportunities, lower customer satisfaction, and other issues. Sales forecasting is the process of determining future sales and the forecasts can be used to maintain the necessary amount of inventory to prevent the under or over-stocking issues. Sales forecasting can affect many company fields such as corporate financial planning, marketing, customer management, etc. Consequently, finding the forecasts and improving them has become one of the important elements of a company [1].

Sales forecasting is a more traditional but still very compelling application of time series forecasting. Time series forecasting is being used as the foundation for the functioning of any process over the time based on the past data. Forecasts are determined using the data from the past and by considering the known factors in the future [3]. Cox and Loomis [2] investigated the advancements in the field of forecasting of the last 25 years by inspecting the books related to the forecasting and thus the advancement of the forecast is noticed. Much effort is dedicated over the past decades for the development and improvement of various forecasting models. Mariana Oliveira and Luis Torgo [15] made an attempt with the ensembles aiming for the improvement of prediction performance of the forecasting. In this research, bagging is compared along with the standard ARIMA and the results show that the approach is promising and can be used as an alternative for forecasting the time series. Random Subspace, Stacking, and Bagging are used for comparison and evaluation of results show that the bagging provides stable improvement for the chosen dataset. RMSE and RAE are used as performance evaluation metrics [16]. In [17] experimented financial time series forecasting by using an intelligent hybrid model is proposed to overcome the issue of capturing the non-stationary property and to identify the accurate movements. In this study, empirical model decomposition (EMD) along with support vector regression (SVR) is introduced and a novel hybrid intelligent prediction algorithm is proposed. Experiment is done using the data collected from the Sina Finance. The outcome of the results shows that EMD-SVR model provides accurate results when compared to individual SVR results. RMSE, MAE, MAPE are used to evaluate the performance of models.

There are more than 10,000 general parts of a truck with different specifications, where some parts are assembled with robots and most of the parts are assembled manually at various workstations. A customer can customize a truck by choosing around twelve thousand different parts that make it about 80-90 percent of a truck. Customers select different parts depending on the various aspects like region, variation in size, etc. It is therefore difficult to determine which components the customers end up choosing. Thus, assessing sales of the components with the use of forecasting plays an important role in optimizing the truck sales process of organizations in overcoming issues like delivery time, stock maintenance, marketing, and discounts, etc. As the parts are large in number, they have been categorized into families like Core 


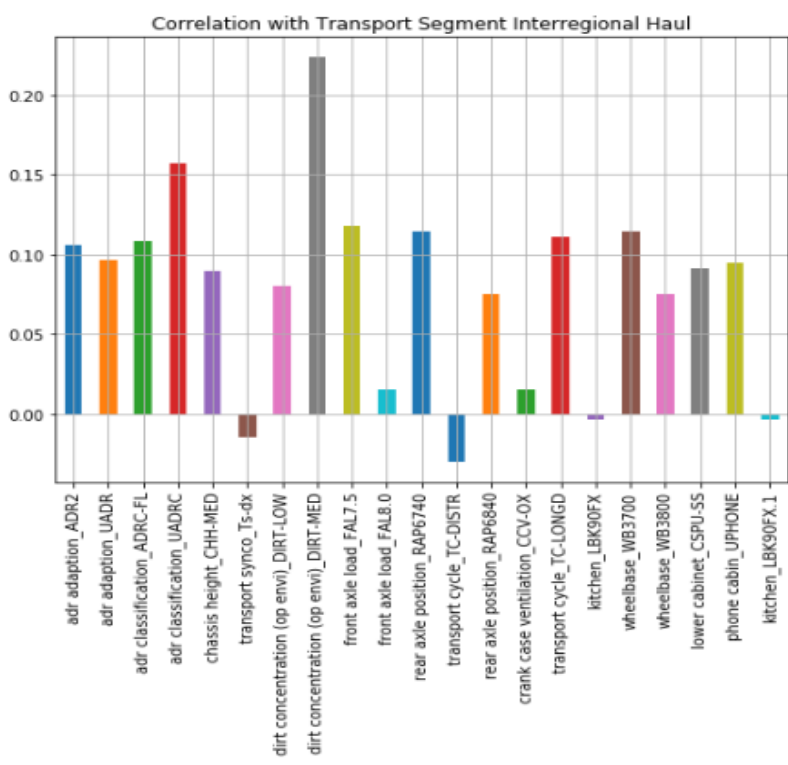

Fig. 1. Correlation with the target Variant.

Components, Rim and Types, Cab interior-driving, etc.

In this work, a sales forecasting model is proposed for truck components in Volvo Trucks Company. In the proposed model, there are three steps which are pre-processing, feature extraction and post-processing. In the pre-processing, a normalization technique is applied to the time-series data to reduce and eliminate the data redundancy. In the second step, feature extraction and selection techniques are used on the normalized data to obtain useful features for the postprocessing step. In the post-processing step, several machine learning methods such as Support Vector Machine Regression, Ridge Regression, Gradient Boosting Regression and Random Forest Regression have been applied to the features of the normalized time-series data. The machine learning methods are trained using the $80 \%$ of data and $20 \%$ is used for testing. Experimental results show that ridge regression model performs well to forecast sales of truck components compared to the other machine learning methods.

\section{TIME-SERIES ForeCASTING}

Sales is considered as a time-series prediction problem. Time series data is categorized into two types. Stationary time series is one whose statistical properties such as the mean, variance and auto-correlation are all constant over time. Non-Stationary time series is the time series whose statistical properties such as the mean, variance and auto-correlation changes over time. Moreover, forecasting using statistical models and machine learning methods are applied in a wide variety of applications such as health [30], economy [33], tourism [26], nuclear magnetic resonance [32], finance [2], [33] etc.

Sales forecasting is an important part of modern business intelligence. Moreover, forecasting plays a major role in decision activities in the areas wing to the essential sales, production, purchasing, finance, and accounting [5]. Sales forecasting is a major issue owing to the unpredictability of demand which relies upon numerous factors [6]. It is a complex problem, especially in the case of a lack of data and noisy data. At present, different time series models such as Holt-Winters, ARIMA, etc, are used [8].

\section{Methodology}

In this work, a forecasting sales model as shown in Fig. 2 is proposed using machine learning methods. The model consists of three different steps which are pre-processing (i.e. normalization), feature extraction and selection and postprocessing.

\section{A. Normalization}

Normalization is the pre-process of the methodology. It rescales the data from original range to a specific scale [18]. Normalization is used to transform the data to a fixed range to eliminate the influence of one data value over the others. In this work, Min-Max Normalization is used which is performed using the following formula.

$$
\hat{x_{n}}=\frac{x_{n}-x_{n}(\min )}{x_{n}(\max )-x_{n}(\min )}
$$

Where $\min$ and $\max$ are the minimum and maximum values in the given data in its range.

\section{B. Feature Extraction and Selection}

Feature extraction and extraction is one of the core concepts which will have a huge influence on the model performance. The data features which are selected for training the machine learning model will have a big impact on the performance because irrelevant features negatively influence the performance of the model. Feature selection and extraction may reduce over fitting and training time as well as improve the accuracy of the model. In this paper, sliding window technique is used to transform the normalized times series data into the features [19]-[21]. Partial Auto Correlation Function (PACF) analysis is performed to obtain the width of the sliding window or lag value [28]. However, before performing this method the time series data must be checked for stationarity. In this paper, Augmented Dickey-Fuller test is used for checking the stationarity which is explained in Section IV.

In Fig. 1, correlation states how the features are related or can be an as mutual statistical dependency between each other or with the chosen target variable. Correlation can be negative (if an increase in one value of a feature decreases the value of the other feature) and positive (if the increase in the one value of the feature increases the other values of the features). If there is no impact between the values of the features then it can be said as no correlation exists [22]. There are different methods for the calculation of correlation coefficient. For instance, Pearson correlation coefficient measures the linear association between the continuous variables and Spearman 


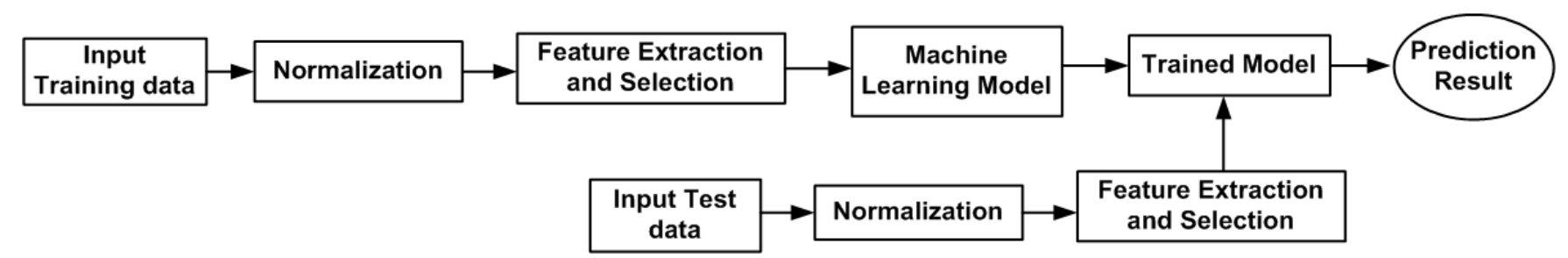

Fig. 2. Proposed forecasting sales model using machine learning methods.

correlation coefficient measures the relationship between the variables by using the monotonic function.

In this work, Spearman Correlation coefficient is used for feature extraction and selection because it is a technique which is used to deal with the problems related to non-linear data [23]. In the proposed method, variables which have a negative correlation with the target variable had been removed to improvise the performance of the machine learning model. Fig. 1 represents the correlation with the feature variants and target variant.

\section{Machine Learning Algorithms}

The utilization of the machine learning regression algorithms for sales forecasting may find better results when compared to the classical time series methods. In this paper, performances of machine learning algorithms such as Support Vector Regression, Random Forest Regression, Ridge Regression, and Gradient Boosting Regression are examined, analysed and discussed.

1) Random Forest Regression: Random Forest (RF) had been proposed by Brieman [7]. It is an ensemble method and a popular statistical based learning technique that extracts useful information from the original samples by forecasting using the bootstrapping method and combining the decision trees in order to perform them. RF regression method takes the mean of the predictions to achieve final results [9]. It can be defined as a kind of additive model which makes predictions by making decisions from various learning models [10]. This can be conventionally described as:

$$
g(x)=f_{0}(x)+f_{1}(x)+f_{2}(x)+\ldots
$$

RF constructs several decision trees and combines them to obtain a better and reliable prediction result. While growing the decision trees, RF adds additional randomness to the model [10].

2) Support Vector Regression: Support vector machine (SVM) is a prominent machine learning model used for classification and regression purposes. SVM consists of two main categories: support vector classification (SVC) and support vector regression (SVR). SVM is a kind of supervised based learning technique which is using a higher level dimensionalfeature-space which produces prediction functions which are broadened on a subset of support vectors [11]. Support Vector Regression is first introduced in 1997 by Vapnik et al. [12].

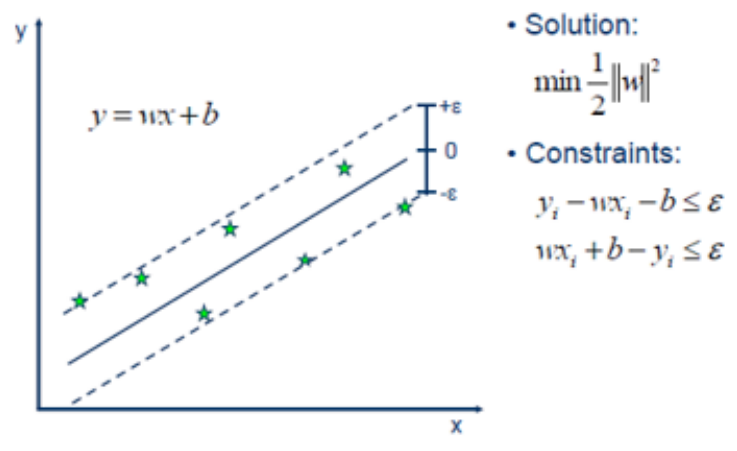

Fig. 3. Support Vector Regression [27].

In Fig. 3, $x_{i}$ exhibits the predictor, $y_{i}$ exhibits dependent variable and $b$ exhibits as the threshold where all the prediction values should be within the range.

3) Ridge Regression: Ridge regression is a method used for the analysis of the multiple regression data that suffers from multi-collinearity [13]. When the occurrence of the multi-collinearity happens, least squares are unbiased but their variances are too large. In this case, by using this method, the optimal prediction result may not be achievable [13]. By the addition of a degree of bias to the regression estimates, the standard error is reduced by the ridge regression. It is expected that the net effect will be to give estimates that are more reliable [13]. The cost function for ridge regression is as follows:

$$
\min \left(\|Y-X(\theta)\|_{2}^{2}+\lambda\|\theta\|_{2}^{2}\right)
$$

Where $X$ is a vector of weights, $(\theta)$ is the coefficient, $(\lambda)$ is denoted by the alpha parameter in the ridge function. So, by altering, the values of the alpha penalty term are controlled. As the alpha value is higher, bigger is the penalty and accordingly coefficients magnitude is reduced. As the parameters shrink, it is mainly used for the multi-collinearity. By doing the coefficient shrinkage, the model complexity is reduced and this process is called regularization.

4) Gradient Boosting Regression: Gradient Boosted Regression trees has been widely used in the prediction problems 
which is one of the most important and powerful machine learning models [14]. The main purpose of using this method is to merge the prediction results from the boosted trees to obtain the final prediction result. Formally it can be described as:

$$
g b r(x)=g_{0}(x)+g_{1}(x)+g_{2}(x)+\ldots
$$

Where the final gradient boosted regression classifier $g b r$ is obtained by adding several classifiers $g_{i}$. Note that, each classifier (e.g. $g_{0}, g_{1}$ etc.) in the model is a decision tree model [14]. Thus, using multiple classifiers in the model provides better predictive performance [14].

Unlike the Random Forest, GBRT uses ensembling-based learning technique named gradient boosting [14]. For a function $f(x)$, assuming the functions is differentiable, gradient descent runs iteratively to find the optimal solution.

$$
x_{t+1}=x_{t}-\left.\eta \frac{\partial f}{\partial x}\right|_{x=x_{t}}
$$

Where $\eta$ is called the step size, $x$ is a vector and the value of $x$ found in the current iteration is its value in the previous iteration added to some fraction of the slope and gradient at this previous value.

Gradient Boosting Trees is an efficient approach to deal with the data with numerical features, data containing categorical features with less than several hundreds of categories. The boosted model can depict the non-linear interaction between the features and the target [14].

\section{Time Series Cross-Validation}

Time Series Cross Validation is a method composed of a set of test sets where each test set contains only one observation. The test sets are averaged to determine the accuracy of the predictions which is often referred to as evaluation on a rolling forecasting origin [25].

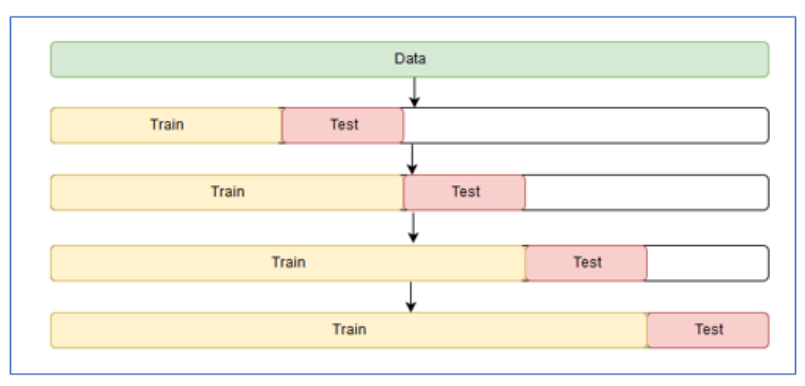

Fig. 4. Time Series Cross Validation

Cross-validation for time series is used because time series consists of the temporal structure and values cannot be randomly mixed in a fold while preserving this structure. With randomization, all-time dependencies between observations will be lost. To overcome this issue, more deceptive approach has to be used where starting with a small subset of data for training purpose, making forecast for the later data points and then checking the accuracy for the forecasted data points.
These data points are then included as part of the next training dataset and subsequent data points are forecasted as shown in Fig. 4. The error obtained on each split is averaged in order to compute a robust estimate of model error [29].

In simpler words, the model has to be trained on a small segment of time series from the beginning until sometime $t$, predictions for the next $t+n$ steps has to be computed, and error needed to be calculated. Then the training sample to be extended to $t+n$ value, predictions from $t+n$ until $t+2 n$ steps has to be computed, and same procedure of moving the test segment of the time series is continued until the last available information. As a result, there will be as may folds as $n$ will fit between the initial training sample and the last observation.

\section{EXPERIMENTAL RESULTS}

\section{A. Dataset Description}

The dataset used in this research consists of the Volvo truck components weekly sales from the year 2014 to 2019 . It consists of 25 components. The final processed data consists of 260 weeks of data. The data is divided into training and testing data, 208 weeks of data is used for training models and 52 weeks data was unused and kept offset for testing. Time Series Split has been used for dividing the training and test set in a continuous manner which has been detailed in the next sections.

Machine learning algorithms have been trained with the dataset using 5-fold time series cross-validation approach where $80 \%$ of the data was used for training and $20 \%$ of the data was used as the test set and the performances have been measured by using the performance metrics which are explained below.

\section{B. Data Quality Check}

In the extracted sales dataset, there are data quality issues and it is necessary to be improved [31]. To ensure the data quality, four dimensions of data quality [36] such as accuracy to remove errors from data, timeliness - to consider up-todate data, consistency - to present the data in same format, completeness - to consider all essential data points related to the sales parameter are considered and assessed in this work.

In this work, we have also used multiple techniques [35] like dropping duplicates, detection of outliers, imputation of missing values, feature selection, data standardization to improve the quality of the dataset [34]. Consequently, based on the data quality assessment techniques which are mentioned above, the used empirical data has a good quality for sales forecasting.

\section{Performance Metrics}

Mean Absolute Error: It is an important approach to measure the forecasting error in the time series analysis. The purpose of using mean absolute error technique is that it measures how close forecast results to the target outcomes. The mean absolute error is estimated by averaging the total 
differences between the forecast results and the target results in the time series problem [24]. Lower the error implies greater the accuracy of the model which is defines as:

$$
M A E=\frac{1}{n} \sum_{i=1}^{n}\left|y_{i}-\hat{y}_{i}\right|
$$

Where ' $n$ ' represents the number of samples, ' $y_{i}$ ' represents actual values and ' $\hat{y}_{i}$ ' represents the predicted or forecasted value.

Root Mean Square Error (RMSE): It is the square root of the mean square error - the root of the average of squared differences between prediction and observation. Lower the error implies greater the accuracy of the model [24] and it is defined as follows:

$$
R M S E=\sqrt{\frac{1}{n} \sum_{i=1}^{n}\left(y_{i}-\hat{y}_{i}\right)^{2}}
$$

Where 'n' represents number of samples, ' $y_{i}$ ' represents actual values and ' $\hat{y}_{i}$ ' represents the predicted or forecasted value.

\section{Stationarity Check}

Stationarity of the dataset has been examined using the augmented dicky fuller test. The below figure represents the results obtained:

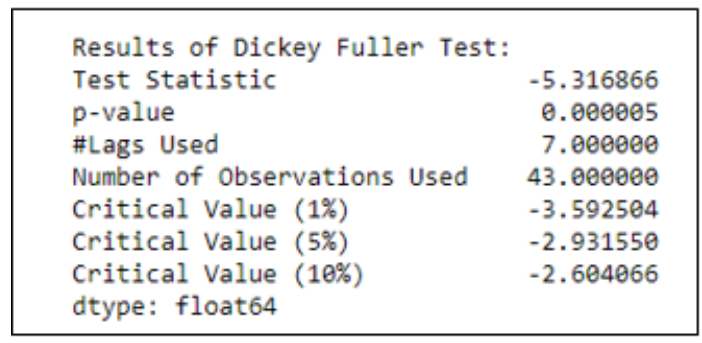

Fig. 5. Augmented Dickey-Fuller Test

From Fig. 5, it can be noted that the p-value is less than 0.5, hence the data is stationary and can be further proceeded for time series analysis. From the PACF analysis, window width for sliding window is chosen as 1 and this lag value is applied to all the feature variables in the data.

\section{E. Gradient Boosting Regressor}

Figures 6, 7, 8 and 9 represent the actual and forecasted sales of target variant obtained using Machine Learning Models where the orange line represents the actual values and blue line represents forecasted sales of the target variant interregional haul.

\section{F. Performance Comparison of Machine Learning Methods for Sales Forecasting}

1) Mean Absolute Error: Fig. 10 (a) represents the Mean Absolute error from the results of the predictions obtained using the Support Vector Regressor, Random Forest Regressor,

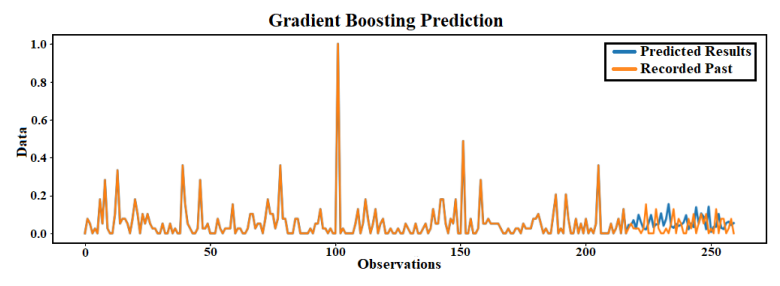

Fig. 6. Gradient Boosting Regression Prediction

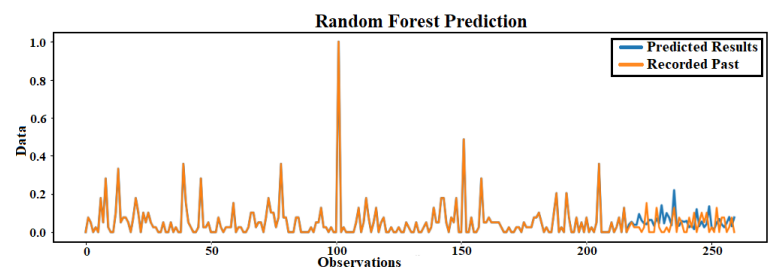

Fig. 7. Random Forest Regressor Prediction

Gradient Boosting Regressor and Ridge Regressor on 5-fold time series cross-validation tests. Based on the results in the figure, it can be seen that except in 2nd fold, Ridge regressor has the least MAE in all the folds and thus can be said as the best-performed algorithm. Gradient boosting has the highest MAE and thus it can be said as the worst performer.

2) Root Mean Square Error: From Fig. 10 (b), it can be noticed that Ridge Regression has shown spectacular performance in terms of results compared to the other algorithms. However, Gradient Boosting Regressor has shown the worst performance with the highest RMSE.

3) Average Mean Absolute Error: From Fig. 10 (c), the average MAE acquired by the Ridge Regression across the 5fold time series cross-validation is 0.058168 , followed by the Random Forest Regressor which is 0.062706 , thereafter SVR which is 0.068752 and finally Gradient Boosting Regressor

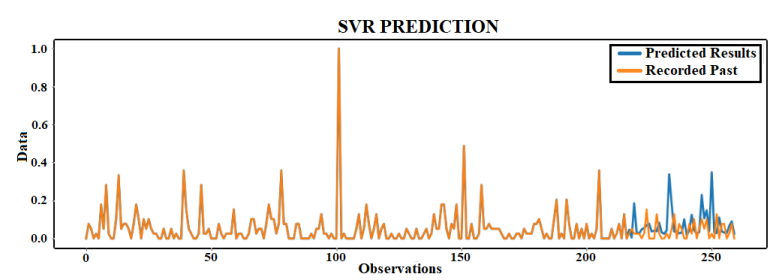

Fig. 8. Support Vector Regressor Prediction

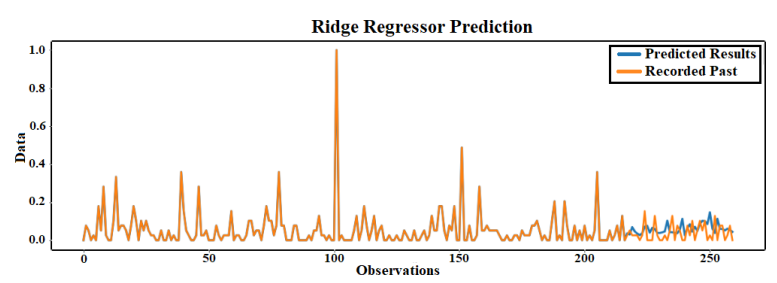

Fig. 9. Ridge Regressor Prediction 


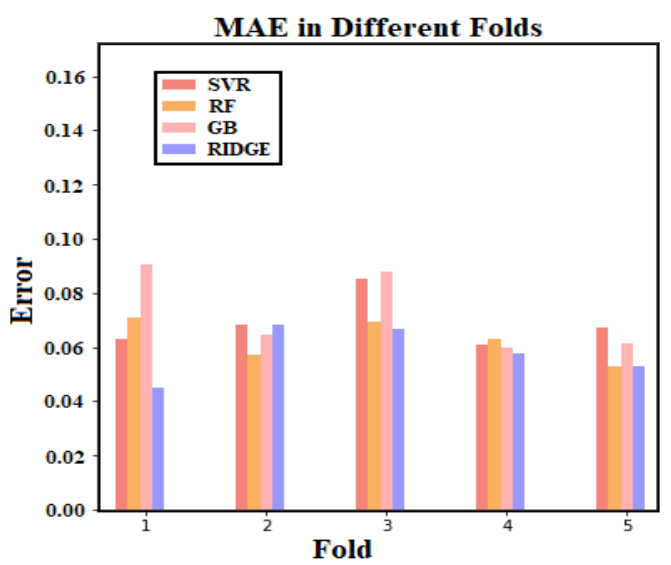

(a)

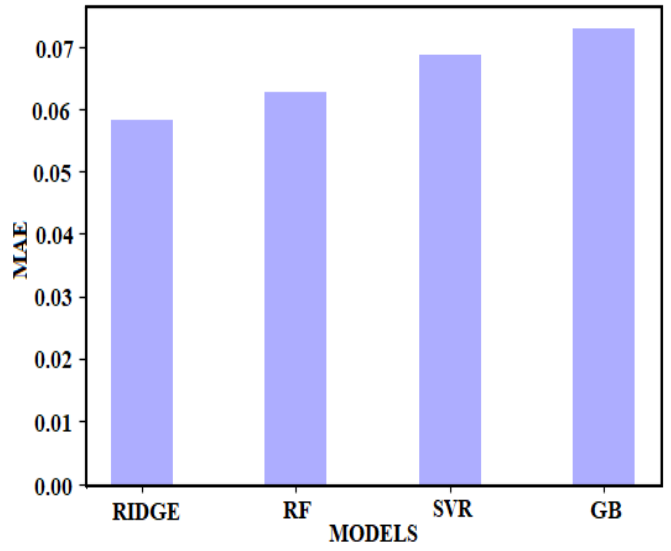

(c)

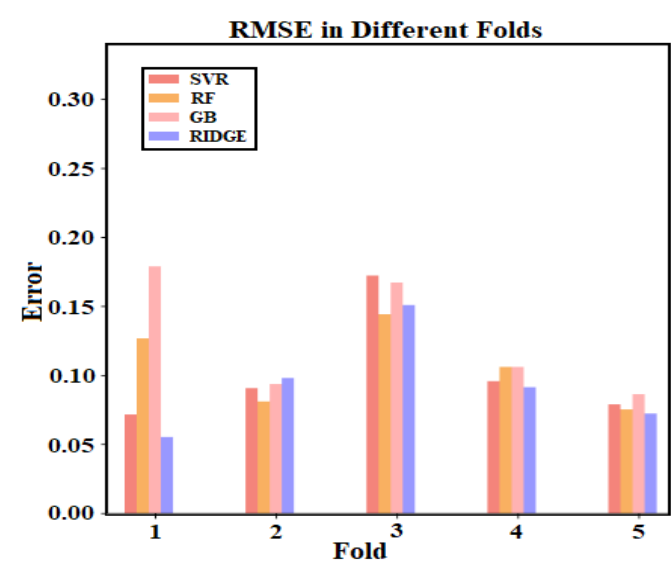

(b)

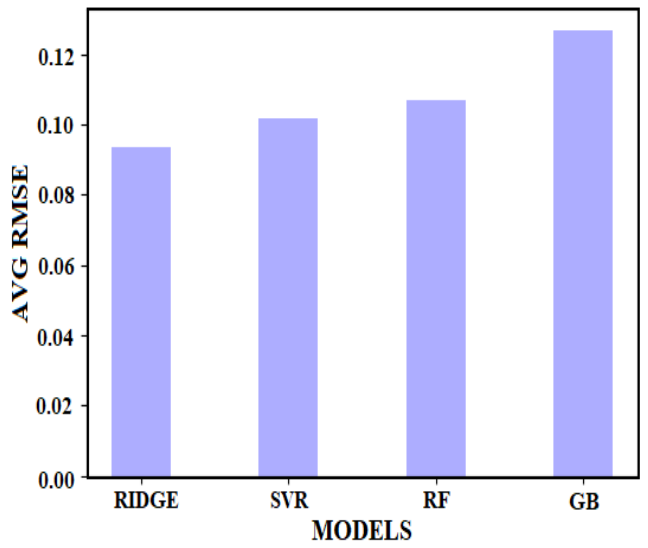

(d)

Fig. 10. Results using different machine learning methods, a) MAE results using the machine learning methods; b) RMSE results using the machine learning methods; c) Average MAE results obtained by regression models on 5-fold time series validation tests; d) Average RMSE results obtained by regression models on 5-fold time series validation tests.

which is 0.072767 . Based on the forecasting results shown in Fig. 10 (c), Ridge Regression provides the best performer with the least error and Gradient boosting is the worst performer with the highest error.

4) Average Root Mean Square Error: From Fig. 10 (d), the average RMSE acquired by the Ridge Regression across the 5 -fold time series cross-validation is 0.09367 , followed by the SVR which is 0.101886 , thereafter Random Forest Regressor which is 0.106836 and finally Gradient Boosting Regressor which is 0.12661 . According to the results, Ridge Regression obtains the best forecasting sales results. However, Gradient boosting is the worst performer with the highest error.

\section{G. Analysis and Discussion}

- Ridge Regression has performed significantly well compared to the other models. This may be because of the regularization approach where the variance is reduced at the cost of some bias initiation which makes it robust to outliers and overfitting.

- Support Vector Regression has performed surprisingly well compared to the random forest and gradient boost- ing, this may be because of its generalization capability. Kernel function for the chosen parameters is set to rbf, other kernel functions like linear, radial may produce better results.

- Random Forest Regressor has not shown good performance, this may be because of overfitting problem which may be preventing from generalizing the model.

- Gradient boosting produced bad results compared to others because of overfitting problem and Gradient Boosting Regressor is harder to tune compared to Random Forest.

\section{CONClusion}

In this paper, forecasting sales of truck components using machine learning methods is proposed. Sales forecasting is a pivotal part of the financial planning of business for any organization and it is important to predict the revenue as well as the profit. Having accurate forecasts enables the company or the organization to explore the possibilities to increase both the revenue and the net profit. In order to achieve good prediction results, various machine learning algorithms such as Random Forest Regressor, Support Vector Regressor, Ridge Regressor, 
and Gradient Boosting Regressor have been used and evaluated on Volvo truck components sales data which can forecast the short term sales and help the organization in making the crucial decisions during their financial planning. After performing the various statistical tests and performance metrics, it is found that Ridge Regression method provides the best performance to predict the future sales for the Volvo truck dataset.

\section{ACKNOWLEDGMENT}

Huseyin Kusetogullari is funded by the research project scalable resource efficient systems for big data analytics by the Knowledge Foundation (Grant: 20140032) in Sweden.

\section{REFERENCES}

[1] C.-J. Lu, T.-S. Lee, C.-M. Lian, "Sales forecasting for computer wholesalers: A comparison of multivariate adaptive regression splines and artificial neural networks," Decision Support Systems, vol. 54, no. 1, pp. 584-596, 2012.

[2] E. J. Jr. Cox, D. G. Loomis, 'Improving forecasting through textbooks A 25 year review," vol. 22, no. 3, International Journal of Forecasting, pp. 617-624, 2006.

[3] M. Gurnani, Y. Korke, P. Shah, S. Udmale, V. Sambhe and S. Bhirud, "Forecasting of sales by using fusion of machine learning techniques," International Conference on Data Management, Analytics and Innovation (ICDMAI), Pune, pp. 93-101, 2017.

[4] C. Chatfield, "Time-Series Forecasting," Chapman and Hall/CRC, 2000

[5] J. T. Mentzer, M. A. Moon, "Sales forecasting management: A demand management approach," International Journal of Forecasting, vol. 22, no. 4, pp. 821-821, 2006

[6] B. M. Pavlyshenko, "Machine-Learning Models for Sales Time Series Forecasting," Journal of Data, vol. 4, no. 1, pp. 1-15, 2019.

[7] L. Breiman, "Random Forests," Machine learning, vol. 45, no. 1, pp. 5-32, 2001.

[8] C. Chatfield, "Chapman \& Hall," CRC, London/Boca Raton, 2001.

[9] J. Huo, T. Shi, J. Chang, "Comparison of Random Forest and SVM for electrical short-term load forecast with different data sources," IEEE International Conference on Software Engineering and Service Science (ICSESS), pp. 1077-1080, 2016.

[10] Turi, "Random Forest Regression," [Online]. Available: https://turi.com/ learn/userguide/supervised-learning/random_forest_regression.html. [Accessed: 21 - May - 2019].

[11] D. Basak , S. Pal , D. C. R. Patranabis, "Support Vector Regression,' Neural Information Processing-Letters and Reviews, vol. 11, no. 10, pp. 203-224, 2007.

[12] V. Vapnik, S. E. Golowich, A. J. Smola, "Support vector method for function approximation, regression estimation and signal processing," NIPS'96: Proceedings of the 9th International Conference on Neural Information Processing Systems, pp. 281 -287, 1997.

[13] J. Al-Jararha, "New Approaches for Choosing the Ridge Parameters," Hacettepe Journal of Mathematics and Statistics, vol. 47, no. 6, pp. 16251633, 2016.

[14] Turi, "Boosted Trees Regression," [Online]. Available: https://turi.com/ learn/userguide/supervised-learning/boosted_trees_regression.html. [Accessed: 21 - May - 2019].

[15] M. R. Oliveira, L. Torgo, "Ensembles for time series forecasting," $A C M L$ JMLR: Workshop and Conference Proceedings, pp. 360-370, 2014.

[16] C. Cheng, W. Xu, J. Wang, "A comparison of ensemble methods in financial market prediction," 2012 Fifth IEEE International Joint Conference on Computational Sciences and Optimization, pp. 755-759, 2012.

[17] F.-J. Lin, "Adding EMD Process and Filtering Analysis to Enhance Performances of ARIMA Model When Time Series Is Measurement Data," Romanian Journal of Economic Forecasting, vol. 18, no. 2, 2015

[18] X. Guo, Y. Pang, G. Yan and T. Qiao, "Time series forecasting based on deep extreme learning machine," 2017 29th Chinese Control And Decision Conference (CCDC), Chongqing, pp. 6151-6156, 2017.

[19] X. Limon, A. Guerra-Hernandez, N. Cruz-Ramirez, H.-G. AcostaMesa, F. Grimaldo, "A Windowing strategy for Distributed Data Mining optimized through GPUs," Pattern Recognition Letters, vol. 93, no. 1, pp. 23-30, 2017.
[20] F. Fumarola, A. Ciampi, A. Appice, D. Malerba, "A Sliding Window Algorithm for Relational Frequent Patterns Mining from Data Streams," International Conference on Discovery Science, pp. 385-392, 2009.

[21] S. Pramod, O. P. Vyas, "Data Stream Mining: A Review on Windowing Approach," Global Journal of Computer Science and Technology Software \& Data Engineering, pp. 1-5, vol. 12, no. 11, 2012.

[22] G. U. Yule, "Why do we sometimes get nonsense-correlations between Time-Series? a study in sampling and the nature of time-series," Journal of the royal statistical society, vol. 89, no. 1, pp. 1-63, 1926.

[23] A. Stuart, "Rank correlation methods," The British Journal of Statistical Psychology, 1956.

[24] L. E. Lehmann, G. Casella, "Theory of point estimation," Springer Science \& Business Media, 2006.

[25] R. J. Hyndman, G. Athanasopoulos, "Forecasting: Principles and Practice," OTexts, 2018.

[26] C. Lim, M. McAleer, "Time Series Forecasts of International Travel Demand for Australia," Tourism Management, vol. 23, no. 4, pp. 389396, 2002.

[27] S. Sayad, "Real time data mining," Self-Help Publishers Cambridge, 2011.

[28] A. Widodo, I. Budi, B. Widjaja, "Automatic lag selection in time series forecasting using multiple kernel learning," International Journal of Machine Learning and Cybernetics, vol. 7, no. 1, pp. 95-110, 2016.

[29] C. Bergmeir, M. J. Benítez, "On the use of cross-validation for time series predictor evaluation," Information Sciences, vol. 191, pp. 192-213, 2012.

[30] S. D. Aaron, A. L. Stephenson, D. W. Cameron, G. A. Whitmore, "A statistical model to predict one-year risk of death in patients with cystic fibrosis," Journal of Clinical Epidemiology, vol. 68, pp. 1336-1345, no. $11,2015$.

[31] W. Dai, K. Yoshigoe, W. Parsley, 'Improving Data Quality Through Deep Learning and Statistical Models," Information Technology - New Generations, Springer International Publishing, pp. 515-522, 2017.

[32] S. C. Connor, M. P. Hodson, S. Ringeissen, B. C. Sweatman, P. J. Mcgill, J. C. Waterfield, J. N. Haselden, ’Development of a multivariate statistical model to predict peroxisome proliferation in the rat, based on urinary $1 \mathrm{H}-$ NMR spectral patterns," Biomarkers, Taylor \& Francis, vol. 9, no. 4-5, pp. 364-385, 2004.

[33] D. Sena, N. K. Nagwani, "Application of time series based prediction model to forecast per capita disposable income," 2015 IEEE International Advance Computing Conference (IACC), pp. 454-457, 2015.

[34] R. Gitzel, "Data Quality in Time Series Data: An Experience Report,' CBI (Industrial Track), pp. 41-49, 2016.

[35] G. Svolba, "Data quality for analytics using SAS", SAS Institute, 2012.

[36] B. T. Hazen, and C. A. Boone, J. D. Ezell, L. A. Jones-Farmer, "Data quality for data science, predictive analytics, and big data in supply chain management: An introduction to the problem and suggestions for research and applications," International Journal of Production Economics, vol. 154, pp. 72-80, 2014. 